\title{
Achatina fulica mucous improves cell viability and increases collagen deposition in UVB-irradiated human fibroblast culture
}

\author{
Nuryana $\mathrm{CT}^{1}$, Haryana $\mathrm{SM}^{2}$, Wirohadidjojo $\mathrm{YW}^{3}$, Arfian $\mathrm{N}^{4}$
}

\begin{abstract}
Introduction: Ultraviolet radiation induces skin photoaging by increasing matrix metalloproteinase-1 (MMP-1). MMP-1 degrades type I and III collagen that comprise the dermal connective tissue. Achatina fulica mucous (AFM) is a natural remedy that has protective effects on fibroblasts and collagen.

Objective: To investigate the effects of AFM on cell viability and collagen deposition in UVB-irradiated human fibroblast culture.

Methods: The mucous was extracted from 50 Achatina fulica snails that were stimulated by a 5-10 Volt electricity shock for 30-60 seconds and converted into powder by the freeze-drying process. The human dermal fibroblast culture was divided into six groups: group 1 were normal fibroblasts without UVB irradiation as normal control, groups $2-5$ consisted of $100 \mathrm{~mJ} / \mathrm{cm}^{2}$ UVB-irradiated fibroblasts. Group 2 had no treatment as negative control, group 3 was treated by PRP $10 \%$ as positive control group and groups 4-6 were treated by various concentrations of AFM $(3.9 ; 15.625$ and $62.5 \mu \mathrm{g} / \mathrm{mL})$. At the end of the experiment, the proliferation was assessed with MTT assay, furthermore collagen deposition was measured by Sirius red assay. Real Time-PCR (RT-PCR) was performed to quantify Coll I, Coll III and MMP-1 mRNA expression, then to measured COL 1/COL III ratio.
\end{abstract}

Results: UVB induced significant lower viability, upregulated MMP-1 and downregulated COL I and COL III mRNA expressions. Meanwhile AFM treated groups demonstrated higher cell viability with downregulation of MMP-1 and upregulation of COL I and COL III mRNA expressions. The ratio of COL I/ III expression was significantly $(p<0.05)$ lower in the AFM treated groups compared to the UVB group. Among AFM treated groups, administration of $62.5 \mu \mathrm{g} / \mathrm{mL}$ AFM represented the best result.

Conclusion: AFM may ameliorate viability of UVB-irradiated human fibroblast culture which associates with downregulating MMP-1, upregulating COL I and Col III, and reducing COL I/III ratio.

Key Words: UVB-irradiated human fibroblast culture; Achatina fulica; Viability; MMP-1; Collagen

\section{Introduction}

Ultraviolet (UV) radiation is the main external factor leading to skin aging. This effect of UV radiation is related to the exposure to specific wavelengths of sunlight. Based on the wavelength, there are several types of UV, which are UVA, UVB, and $\mathrm{UVC}^{[1]}$. UVC radiation with $200-290 \mathrm{~nm}$ in wavelength is reflected by the ozone layer, so its impact on the skin is not significant. The shorter wavelengths have greater impact. UVB with a wavelength of $290-320 \mathrm{~nm}$ provides more energy than UVA which has a wavelength of $320-400 \mathrm{~nm}$. UVB is able to penetrate the epidermis and dermis. UVB exposure is linked to a higher non-melanoma skin cancer (NMSC) risk compared to UVA. Increasing UVB radiation on the earth induces inflammatory responses, apoptosis, and subsequent skin damage, such as photoaging ${ }^{[2]}$.

The characteristics of photoaging are wrinkles, loss of elasticity, dryness, frailty, rough skin texture, telangiectasias and pigmentation disorders that indicate structural and functional alteration of extracellular matrix $(\mathrm{ECM})^{[3]}$. The main component of ECM is collagen that forms the basic framework of connective tissue. The dermal collagen particularly consists of collagen type $I^{[4]}$. The type $I$ collagen is a heterotrimer molecule characterized by the triple helix with three related $\alpha$ chains. It indicates mature collagen, forms thick fibers and maintains high tensile strength. The least amount of dermal collagen is collagen type III consisting of a supercoiled $\alpha 1$ (III) chains that forms a homotrimer molecule ${ }^{[5]}$. Collagen III has thin fibers with low tensile strength and is also called embryonic or immature collagen ${ }^{[6]}$. As age increases, collagen decreases by about $1 \% /$ year. Decreased type I and III collagen along with an elevation of the type I/III collagen ratio are indicators of photoaging $\operatorname{skin}^{[7]}$. Collagen degradation is caused by MMP-1 due to increasing reactive oxygen stress (ROS)-induced by UVB exposure ${ }^{[8]}$.

Current research has focused to discover anti-aging agents from natural ingredients, plant products, and herbal extracts ${ }^{[9]}$. Achatina fulica mucous (AFM) is a natural remedy containing anti-aging agents that have been used in ancient medicine. Previous study revealed that AFM contains Achasin, a broad spectrum antibiotic and anti-inflammation agent ${ }^{[10]}$. Moreover, AFM also contains 
Acharan sulfate which is a glycosaminoglycan ${ }^{[11]}$, the trace mineral, copper which contributes to collagen synthesis, and vitamins A and E as antioxidants ${ }^{[12,13]}$.

Accordingly, this experiment aimed to determine how the AFM, which is rich in antioxidants, anti-inflammation activity, and glycosaminoglycans can be turned into high end-value products. In this paper, we observed the protective effects AFM on UVB-induced fibroblast photoaging by assessing collagen deposition, MMP-1 expression and the altered ratio of type I/III collagen expression.

\section{Materials and Methods}

This in vitro research with post-test only control group design had obtained approval from the ethics committee of the Faculty of Medicine, Public Health and Nursing, Universitas Gadjah Mada (Ref: $\mathrm{KE} / \mathrm{FK} / 0682 / \mathrm{EC} / 2018$ ).

\section{Snails mucous and materials}

AFM was collected by triggering the mucous secretion from 50 Achatina fulica snails using 5-10V electricity shock for 30-60 seconds. The mucous was stored in a sterilized jar before converted into powder by freeze-drying process ${ }^{[12]}$. Platelet-rich plasma (PRP) was purchased from the Dermato-Venerology Department of Medicine, Public Health and Nursing Faculty, Universitas Gadjah Mada, Yogyakarta, Indonesia.

\section{Cell Culture}

We cultured normal human dermal fibroblasts (NHDFs) to be used for experiments at passages $>4$ from 11-13 years old boys' foreskins. NHDFs were maintained using Dulbecco's Modified Eagle's Medium (DMEM; Sigma-Aldrich Corporation, St. Louis, MO, USA) which were supplemented with $10 \%$ heat in activated fetal bovine serum (FBS), 100 units $/ \mathrm{ml}$ Penicillin, and $100 \mu \mathrm{g} / \mathrm{ml}$ Streptomycin (Penstrep-Gibco; Invitrogen Corporation, Carlsbad, CA, USA) in the humidity of $5 \% \mathrm{CO}_{2}$ at $37^{\circ} \mathrm{C}$. Subculture procedure using trypsin was performed in the fibroblasts. Briefly, after rinsing with phosphatebuffered saline (PBS), cells were detached using trypsin and were cultured with completely fresh growth medium.

\section{UVB irradiation and treatments}

UVB irradiation procedure was done in the NHDFs model. After 80$90 \%$ confluence, the NHDFs were rinsed with PBS and then exposed to UVB light in fresh PBS-filled wells with a bank of six UVB lamps (Philips UVB TL 40W/12RS; LIPI, Jakarta, Indonesia) at a dose of $100 \mathrm{~mJ} / \mathrm{cm}^{2}$ for 330 seconds. Then, the NHDFs were rinsed with PBS three times and immediately treated with AFM in $3.9 \mu \mathrm{g} / \mathrm{mL}$, $15.625 \mu \mathrm{g} / \mathrm{mL}$ and $62.5 \mu \mathrm{g} / \mathrm{mL}$ concentrations. Normal controls consisted of the NHDFs that had no treatment and were not exposed to UVB irradiation, and negative control were NHDFs without any treatment and were exposed to UVB radiation, while positive control was the NHDFs that were exposed to UVB radiation with $10 \%$ PRP treatment. Then, the NHDFs were incubated for 72 hours.

\section{NHDFs viability procedure using MTT assay}

We used MTT (3-(4, 5-dimethylthiazolyl-2)-2, 5-diphenyltetrazolium bromide) assay procedure to examine NHDFs viability by monitoring color changes during reduction of tetrazolium salts. Ten microliters of the MTT reagent were added to every well after 72 hours. Then, the NHDFs were incubated in humidity with atmosphere $\left(5 \% \mathrm{CO}_{2}\right.$ at $37^{\circ} \mathrm{C}$ ) for 2 hours. Next, the NHDFs were viewed under an inverted microscope. When the intracellular purple formazan crystals were clearly visible, the substrate-containing medium was removed, and
$100 \mu \mathrm{L}$ of DMSO were added to each well. The optical density (OD) was read using a microplate absorbance reader (Biorad iMark ${ }^{\mathrm{TM}}$; Bio-Rad Laboratories, Singapore) at $\lambda 570 \mathrm{~nm}$.

\section{Measurement of collagen deposition by Sirius red}

The Sirius red staining procedure was done to quantify collagen deposition in the NHDFs. The NHDFs were rinsed with PBS. Furthermore, at room temperature NHDFs were fixed with Bouin's solution for 60 minutes. The solvent was eliminated and plates were rinsed in running tap water for 15 minutes. The 96-well plates were air-dried overnight, after that $200 \mu \mathrm{L}$ Picro-Sirius Red dye $(1 \mathrm{mg} / \mathrm{mL}$ in picric acid) was added to every well for 60 minutes with light shaking. In each well, unbound dye was removed by rinsing thrice with $0.01 \mathrm{~N} \mathrm{HCl}$ while the bound dye was dissolved with $100 \mu \mathrm{L} 0.5 \mathrm{~N} \mathrm{NaOH}$ with light shaking at room temperature for 30 minutes. Collagen-dye optical density (OD) was read with a microplate absorbance reader (Biorad iMark ${ }^{\mathrm{TM}}$; Bio-Rad Laboratories, Singapore) at $\lambda 540 \mathrm{~nm}$.

\section{Measurement of MMP-1, COL I, and COL III mRNA expresión}

Quantitative real-time PCR (qRT-PCR) was conducted to quantify the mRNA expression of MMP-1, COL I, and COL III. The levels of mRNA expression were normalized with GAPDH as the reference gene. Briefly, RNA was extracted from the NHDFs by miRCURY Cell And Plant Isolation Kit (Exiqon; Lot\# 32213). cDNA was synthesized using miRCURY LNA Universal RT microRNA PCR Universal cDNA Synthesis Kit II, 8-64 rxns (Exiqon; Lot \# 629693). RNA was quantified with a nanodrop using NanoVue Plus spectrophotometer (Biochrom, a division of Harvard Bioscience, Inc., Harvard, USA). Quantitative PCR was conducted using protocol from Applied Biosystem 7500 FAST real-time PCR using Toyobo thunderbird ${ }^{\circledR}$ SYBR ${ }^{\circledR}$ qPCR Mix (cat\#QPS-201). The qRT-PCR was done with the following conditions: initial denaturation at $95^{\circ} \mathrm{C}$ for $2 \mathrm{~min}$, followed by 40 cycles for denaturation at $95^{\circ} \mathrm{C}$ for $10 \mathrm{sec}$., annealing at $58^{\circ} \mathrm{C}$ for $15 \mathrm{sec}$., and extension at $72^{\circ} \mathrm{C}$ for $20 \mathrm{sec}$. The $2-\Delta \Delta \mathrm{Cq}$ method was used to assess the mRNA expression of MMP, COL I, and COL III.

The following primers were used:

MMP1: forward CTGAAAGTGACTGGGAAACC and reverse GACAAACTGAGCCACATCAG,

COL I: forward GTGCTAAAGGTGCCAATGGT and reverse GTGGGGAATGGCAAGCAAAA;

COL III: forward CCAGGAGCTAACGGTCTCAG and reverse CAGGGTTTCCATCTCTTCCA,

GAPDH: forward GGAGCGAGATCCCTCCAAAAT and reverse GGCTGTTGTCATACTTCTCATGG.

Each reaction was run in triplicate.

\section{Statistical Analysis}

The results are stated in average \pm standard error of measurement (SEM). One-way ANOVA was done to determine any significant differences between groups followed by LSD test for the Post-hoc multiple comparison test. The degree of statistical significance was set at $\mathrm{p}<0.05$. The SPSS 22 was performed to assess statistical analyses (IBM Corporation, Chicago, USA).

\section{Results}

\section{AFM increased fibroblast viability}

UVB exposure induces cell death in normal human dermal fibroblasts (NDHFs). In order to evaluate the viability of the AFM, the NDHFs were treated with serial concentrations $(0.49,0.98,1.95$, 


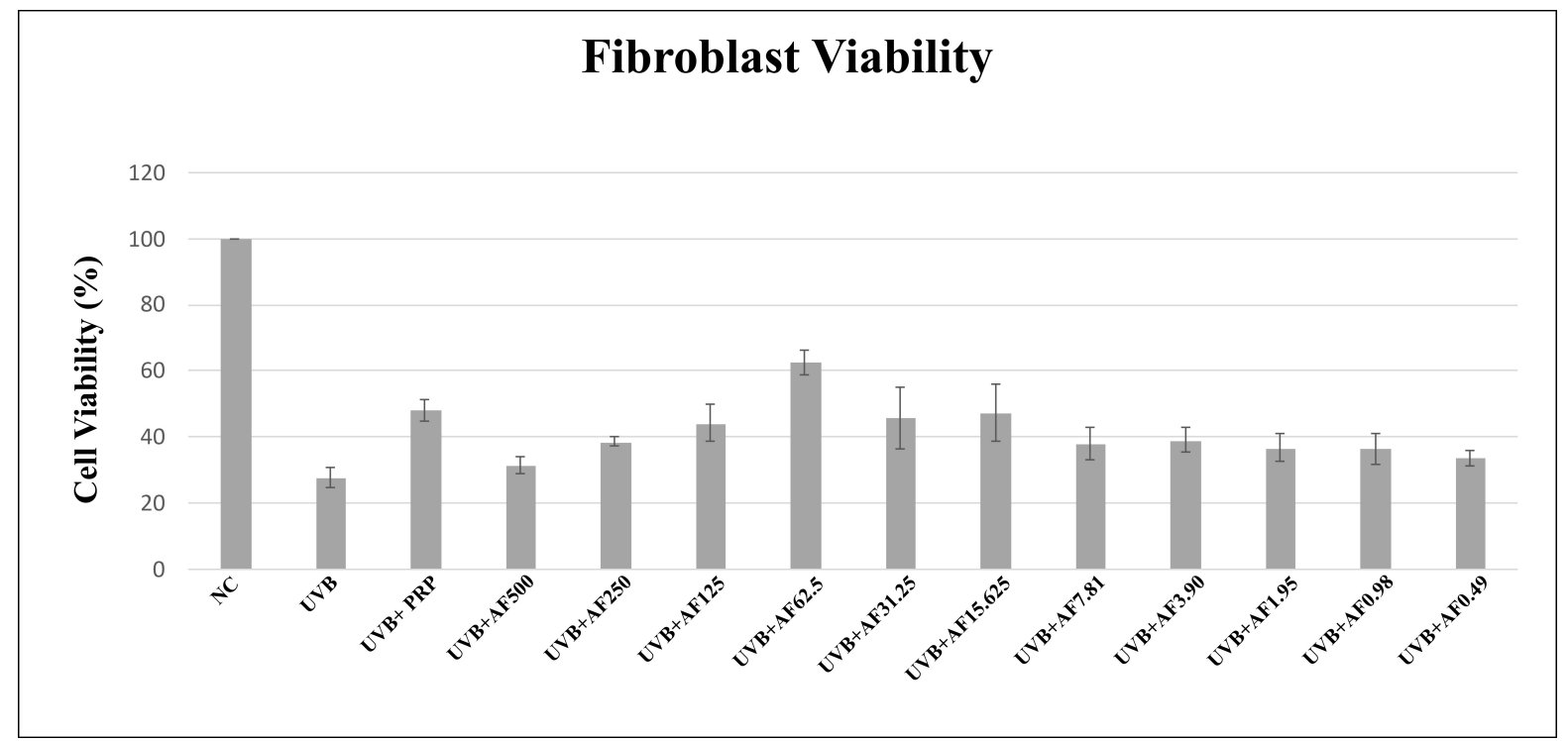

Figure 1. Fibroblast viability test of AFM in NDHFs. NDHFs were cultured in DMEM until 80-90\% confluence and exposed to $100 \mathrm{~mJ} / \mathrm{cm} 2 \mathrm{ultraviolet} B$ (UVB). Cells were added with or without serial concentrations of $A F M$ for $72 \mathrm{~h}$, and afterward the MTT assay was conducted. All data are counted as the average $\pm S E M$ of triplicate experiments. Normal control (NC) was NDHFs not exposed to UVB irradiation and received no treatment, UVB was NDHFs exposed to UVB irradiation and received no treatment, UVB +PRP was NDHFs exposed to UVB irradiation and received treatment with PRP $10 \%$, and UVB+AF was NDHFs exposed to UVB irradiation and received treatment with various concentrations ( $\mu \mathrm{g} / \mathrm{mL}$ ) of AFM.

$3.90,7.81,15.625,31.25,62.5,125,250$, and $500 \mu \mathrm{g} / \mathrm{mL})$ of AFM for 72 hours after exposure to UVB $\left(100 \mathrm{~mJ} / \mathrm{cm}^{2}\right)$. The viability of NHDFs were quantified using an MTT assay (as described in the methods). The AFM viability increased up to $62.5 \mu \mathrm{g} / \mathrm{mL}$ concentration but showed slight reduction at $125 \mu \mathrm{g} / \mathrm{mL}$ concentration (Figure 1). Therefore, three different concentrations $(3.9 \mu \mathrm{g} / \mathrm{mL}$, $15.625 \mu \mathrm{g} / \mathrm{mL}$ and $62.5 \mu \mathrm{g} / \mathrm{mL}$ ) of AFM were chosen to test in this study. As a positive control, PRP had a viability of 2 folds higher than UVB as a negative control. Compared to PRP, the AFM viability of NHDF $15.625 \mu \mathrm{g} / \mathrm{mL}$ was not significantly different $(p>0.05)$ but the
AFM viability of NHDF $62.5 \mu \mathrm{g} / \mathrm{mL}$ was significantly $(\mathrm{p}<0.05)$ higher whereas AFM $3.9 \mu \mathrm{g} / \mathrm{mL}$ was significantly $(\mathrm{p}<0.05)$ lower.

\section{AFM downregulated MMP-1 mRNA expression}

UV exposure increases MMP-1 mRNA expression which triggers degradation of fibrillar collagen, especially type I and III collagen in the dermis. To observe the effects of AFM on UVB-mediated mRNA expression of MMP-1, the mRNA levels were quantified by qRT-PCR. After UVB-irradiation, the NDHFs were incubated for

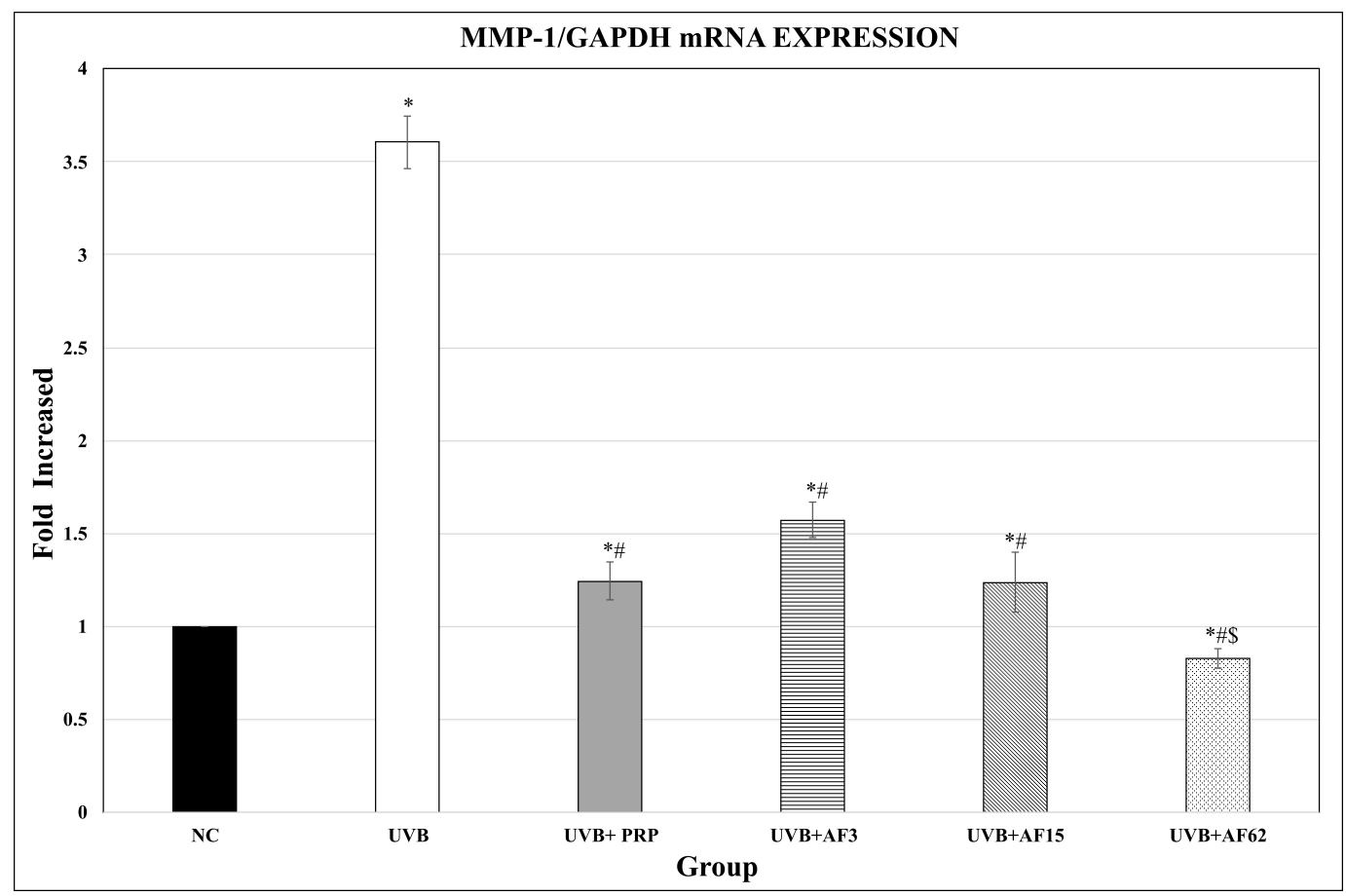

Figure. 2. Quantitative PCR (qPCR) examination of MMP-1 mRNA expression. NHDFs were induced with UVB 100mJ/cm 2 and administered with various concentrations of AFM $(3.9 \mu \mathrm{g} / \mathrm{mL} ; 15.625 \mu \mathrm{g} / \mathrm{mL}$ and $62.5 \mu \mathrm{g} / \mathrm{mL})$. The value is provided as the mean $\pm S E M . * p<0.05$ indicates the significant versus the normal control. \#p $<0.05$ indicates the significant versus a UVB. $\$ p<0.05$ indicates the significant versus PRP. Normal control (NC) was NDHFs not exposed to UVB irradiation and received no treatment, UVB was NDHFs exposed to UVB irradiation and received no treatment, UVB+PRP was NDHFs exposed to UVB irradiation and received treatment with PRP $10 \%$, and UVB $+A F$ was NDHFs exposed to UVB irradiation and received treatment with various concentrations $(3.9 \mu \mathrm{g} / \mathrm{mL} ; 15.625 \mu \mathrm{g} / \mathrm{mL}$ and $62.5 \mu \mathrm{g} / \mathrm{mL})$ of $\mathrm{AFM}$. 
72 hours with $3.9 \mu \mathrm{g} / \mathrm{mL}, 15.625 \mu \mathrm{g} / \mathrm{mL}$ and $62.5 \mu \mathrm{g} / \mathrm{mL}$ concentrations of AFM. As shown in Figure 2, UVB induction in the UVB group demonstrated significantly higher MMP-1 mRNA expression compared to the normal control group (NC). Significantly lower mRNA expression of MMP-1 was shown in the PRP and AFM treated groups. In the AFM group, we found significant differences between AF62 compared to AF3 and AF15, but there was no significant difference between AF3 and AF15.AFM increased collagen deposition, COL I and COL III mRNA expressions with reduced COL I/III ratio.

The in vitro effect of AFM on NHDFs collagen deposition was quantified by Sirius red dye binding and spectrophotometry. Incubation of NHDFs for 72 hours with various concentration of AFM after irradiation with $100 \mathrm{~mJ} / \mathrm{cm}^{2}$ UVB induced an enhancement in the amount of NHDFs collagen deposition (Figure 3A). The red stainings represent collagen deposition. In the UVB group, collagen deposition was lower than the normal control group $(p<0.05)$. Moreover, collagen deposition arrangements were more compact and regular in the AFM groups (Figure 3A). The highest concentration of AFM $(62.5 \mu \mathrm{g} / \mathrm{mL})$ exhibited significantly $(p<0.05)$ higher collagen deposition. A confirmatory experiment assessed the type of collagen by qRT-PCR examination of COL I, COL III, and COL I/ III ratio (Figure 3B).

\section{Discussion}

This experiment highlighted the molecular mechanism of AFM for increasing cell viability and collagen deposition in UVB-induced human fibroblast culture through alteration of the MMP-1, and ratio of type I/III collagen mRNA expression. UVB irradiation (290-320 $\mathrm{nm}$ ) leads to increased cell death in $\mathrm{NDHFs}^{[13]}$. In this study, the NDHFs viability in the UVB group decreased compared to the other groups. This finding indicated that the dose of UVB irradiation was adequate to conduct irradiation procedures in line with the previous study. UVB activates cytokines productions that affect cellular mitosis, apoptosis, and cell death. UVB induces the generation of ROS including superoxide anion (O2-) or hydrogen peroxide $(\mathrm{H} 2 \mathrm{O} 2)^{[14]}$. In this study, we found an improvement of NDHFs viability in the AFM groups compared to UVB groups (Figure 1). AFM contains vitamin A and E (data not shown) as antioxidants that will directly react with ROS. ROS triggers the expression of pro-inflammatory cytokines production. Interestingly, AFM contains Achasin that had been reported to have anti-inflammatory effects on the $\operatorname{skin}^{[15]}$.

Increasing of ROS induced by UVB stimulates the synthesis of MMP-1. ROS activates MAP kinases which initiates activator protein 1 (AP-1). AP-1 performs an important role in the transcriptional regulation of MMP-1 which triggers an enhancement of MMP- $1^{[8]}$. MMP-1 is the main protease that is competent to initiate fragmentation of fibrillar collagen in the skin, especially types I and III collagen, while other types of MMP further break down degraded collagen fragments. Collagen is arranged by repeated triple helix that is stabilized by cross linking to other molecules. MMP-1 breaks collagen fibrils at the centers of the triple helix. Therefore, an increase in MMP-1 causes the accumulation of collagen fragments and damages the structure and function of the $\mathrm{ECM}^{[16]}$. Our study revealed upregulation of MMP-1 mRNA expression after UVB exposure (Figure 2), while AFM treatment

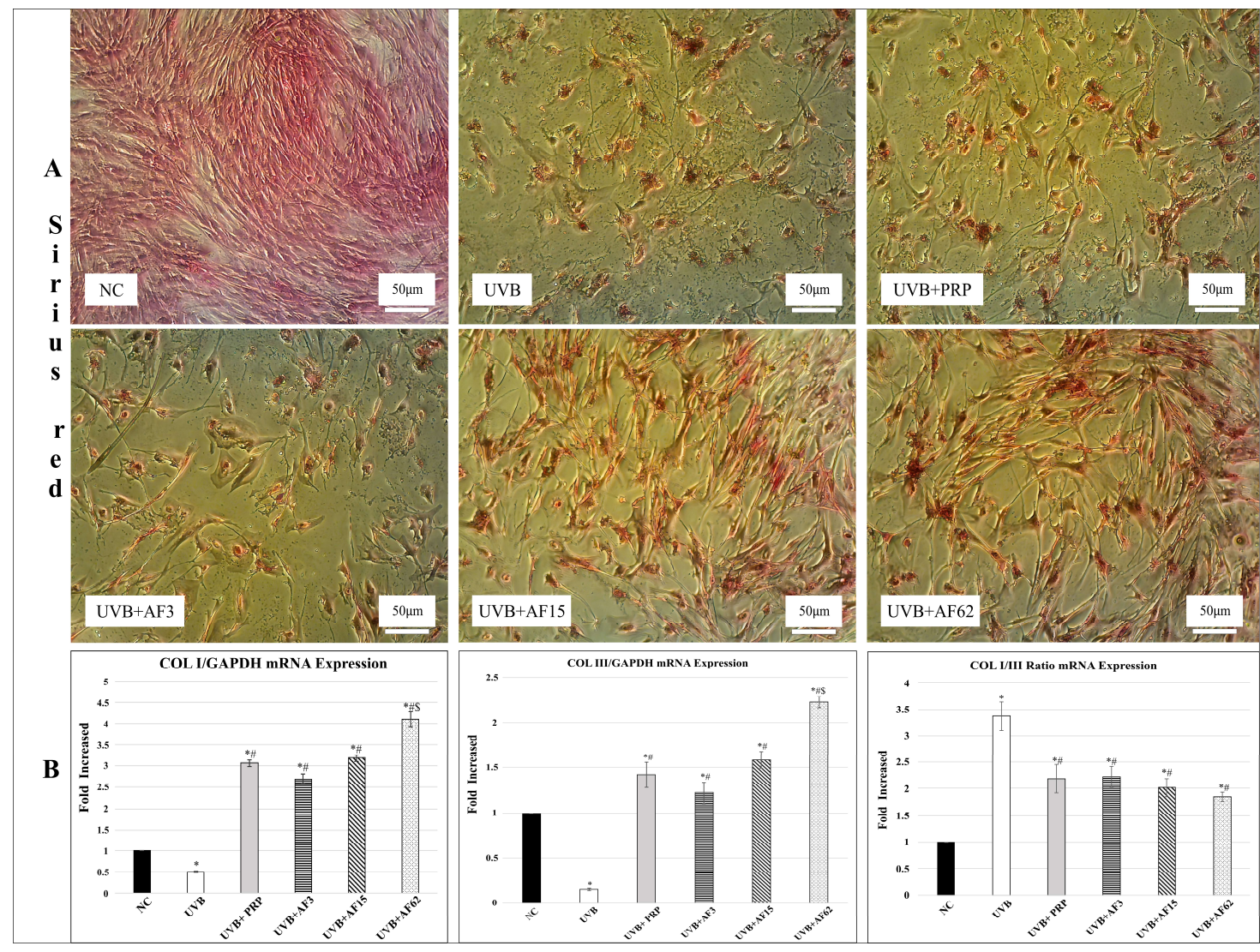

Figure 3. A. Representative pictures of Sirius red staining for collagen deposition. Positive staining was reddish shown. B. Quantitative PCR (qPCR) interpretation of COL I, COL III and COL IIIII ratio mRNA expressions. UVB+AF62 group demonstrated significantly higher COL I and COL III mRNA expressions compared with the NC group. UVB group demonstrated significantly lower expressions of both COL I and COL III mRNA compared with the NC group. ${ }^{*} p<0.05$ indicates the significant versus the normal control. \#p $<$ 0.05 indicates the significant versus a UVB. $\$ p<0.05$ indicates the significant versus PRP. 
downregulates the MMP-1 mRNA expression. Compared to PRP as a positive control, AFM has greater effects in downregulation of MMP-1. Previous study reported that PRP is a potent anti-aging material capable of inhibiting MMP-1 mRNA expression and enhancing collagen synthesis ${ }^{[17]}$.

Collagen, synthesized by fibroblasts, is the major constituent of ECM. It constitiutes the basic structure and covers $70-80 \%$ of the connective tissue dry weight. Characteristic of the structure is the repeated triple helix that helps collagen to form cross-links with other molecules to maintain mechanical integrity and tensile strength. Increasing MMP-1 due to UVB irradiation induces fragmented collagen which downregulates new collagen synthesis and collagen deposition ${ }^{[18]}$. This previous finding is parallel with the histological appearance in this study in Figure 3A that shows UVB reduced collagen deposition while AFM treatment significantly promoted the collagen deposition in NHDFs, which was evidenced by the Sirius red collagen staining.

Commonly, the collagens found in the skin are collagen type I and III Type I collagen accounts for approximately $85 \%-90 \%$ from the total fibrillar collagen in the skin. Collagen I is mature collagen, which has thick fibers and high tensile strength. Previous study revealed that type I collagen synthesis decreases in photoaged skin ${ }^{[19]}$. Our study confirmed that AFM upregulates COL I mRNA expressions. As shown in Figure 3B, AFM increased type I collagen which was reconfirmed by the quantification of COL I mRNA expression in UVB-induced NHDFs. Increasing COL I mRNA expression indicates increasing type I collagen synthesis.

The lesser amount, approximately $10 \%$, of dermal collagen is type III collagen. It has a particular assignment in early organ development as embryonic collagen. The type III collagen is immature collagen which has a thin bundles and low tensile strength. The number of type III collagen is highest in fetal development and then decreases with age. Complex alterations in the structure of collagen type III lead to decreasing dermal stability ${ }^{[20]}$. Our study confirmed AFM upregulates COL I and COL III mRNA expressions which were associated with higher collagen deposition based on Sirius Red quantification. The ratio between COL I and COL III was also reduced in our study which showed similarity with younger age collagen content. This finding was reconfirmed by the quantification of COL III mRNA expression in UVB-induced NHDFs as shown in Figure 3B.

In adult human skin, types I and III collagen account of $80-85 \%$ and $10-15 \%$, respectively. During skin formation, collagen types undergo fluctuations in relative ratio during aging ${ }^{[21]}$. In the fetus, the type I/III collagen ratio is 1 and subsequently increases with age in adolescents, adult and elderly to $2.27,2.46$ and 2.97 , respectively ${ }^{[7]}$. The elevation of type I/III collagen mRNA expression ratio in aged skin is consistent with the results in this study as exhibited in Figure 3B. Even though the level of COL I and COL III mRNA expressions in AFM increased, the type I/III collagen mRNA expression ratio level was decreased. These findings indicate AFM stimulates collagen synthesis. Some limitations of this study are we did not quantify the ROS and other senescence markers such as Senescence Associated- $\beta$ Galactosidase (SA- $\beta \mathrm{Gal})$, p21 or p16 but focused on downregulation of MMP-1 and increases in cell viability and collagen deposition.

\section{Conclusion}

In this study, we demonstrated the protective effects of AFM on UVB-irradiated human fibroblast culture through increasing cell viability, collagen deposition, COL I and COL III mRNA expressions along with reducing MMP-1 expression.

\section{References}

1. Cavinato M, Jansen-Dürr P. Molecular mechanisms of UVBinduced senescence of dermal fibroblasts and its relevance for photoaging of the human skin. Exp Gerontol. 2017;94:78-82.

2. Bosch R, Philips N, Suárez-Pérez J, Juarranz A, Devmurari A Chalensouk-Khaosaat $\mathrm{J}$, et al. Mechanisms of photoaging and cutaneous photocarcinogenesis, and photoprotective strategies with phytochemicals. Antioxidants. 2015;4(2):248-68.

3. Scharffetter-Kochanek K, Brenneisen P, Wenk J, Herrmann G, $\mathrm{Ma}$ W, Kuhr L, et al. Photoaging of the skin from phenotype to mechanisms. Exp Gerontol. 2000;35(3):307-316.

4. Tigges J, Krutmann J, Fritsche E, Haendeler J, Schaal H, Fischer JW, et al. The hallmarks of fibroblast ageing. Mech Ageing Dev. 2014;138:26-44

5. Peng Y, Song X, Zheng Y, Wang X, Lai W. Circular RNA profiling reveals that circCOL3A1-859267 regulate type I collagen expression in photoaged human dermal fibroblasts. Biochem Biophys Res Commun. 2017;486(2):277-84.

6. Kielty CM, Grant ME. The Collagen Family: Structure, Assembly, and Organization in the Extracellular Matrix. In: Royce PM, Steinmann B, editors. Connective Tissue and its Heritable Disorders [Internet]. Hoboken, NJ, USA: John Wiley \& Sons, Inc.; 2002 [cited 2019 Sep 28]. p. 159-221. Available from: http://doi.wiley.com/10.1002/0471221929.ch2

7. Cheng W, Yan-hua R, Fang-gang N, Guo-an Z. The content and ratio of type I and III collagen in skin differ with age and injury. Afri J Biotechnol. 2011;10(130):6.

8. van Marion MM, Baaijens FP, Mol A, Merkx M, Rubbens MP Matrix metalloproteinases and collagen remodeling A Literature Review. 2006;

9. Mahawar M, Jaroli D. Traditional zootherapeutic studies in India: a review. J Ethnobiol Ethnomedicine. 2008;4(1):17.

10. Biswas C, Sinha D, Mandal C. Investigation on interaction of Achatinin, a 9-O-acetyl sialic acid-binding lectin, with lipopolysaccharide in the innate immunity of Achatina fulica snails. Mol Immunol. 2000;37(12):745-754.

11. Liu J, Zhou L, He Z, Gao N, Shang F, Xu J, et al. Structural analysis and biological activity of a highly regular glycosaminoglycan from Achatina fulica. Carbohydr Polym 2018;181:433-41.

12. Berniyanti T, Waskito EB, Suwarno S. Biochemival characterization of an antibactrial glycoprotein from Achatina fulica ferussac snail mucus local isolate and their implication on bacterial dental infection. Indones J Biotechnol [Internet]. 2015 [cited 2017 May 14];12(1). Available from: https://journal.ugm.ac.id/ijbiotech/article/download/7765/6015

13. Amaro-Ortiz A, Yan B, D'Orazio J. Ultraviolet radiation, aging and the skin: prevention of damage by topical cAMP manipulation. Molecules. 2014;19(5):6202-19.

14. Masaki H, Izutsu Y, Yahagi S, Okano Y. Reactive oxygen species in $\mathrm{HaCaT}$ keratinocytes after UVB irradiation are triggered by intracellular $\mathrm{Ca} 2+$ levels. J Investig Dermatol Symp Proc. 2009;14(1):50-2.

15. de la Secreción P. Assessment of antimicrobial activity and healing potential of mucous secretion of Achatina fulica. Int $\mathrm{J}$ Morphol. 2012;30(2):365-373.

16. Quan T, Qin Z, Xia W, Shao Y, Voorhees JJ, Fisher GJ. Matrixdegrading metalloproteinases in photoaging. J Investig Dermatol Symp Proc. 2009;14(1):20-4.

17. Cho JM, Lee YH, Baek R-M, Lee SW. Effect of platelet-rich plasma on ultraviolet b-induced skin wrinkles in nude mice. J Plast Reconstr Aesthet Surg. 2011;64(2):e31-9.

18. Makpol S, Jam FA, Yusof YAM, Wan Ngah WZ. Modulation of collagen synthesis and its gene expression in human skin fibroblasts by tocotrienol-rich fraction. Arch Med Sci. 2011;5:889-95.

19. Yano H, Hamanaka R, Nakamura M, Sumiyoshi H, Matsuo N Yoshioka H. Smad, but not MAPK, pathway mediates the expression of type I collagen in radiation induced fibrosis. Biochem Biophys Res Commun. 2012;418(3):457-63. 
20. Naffa R, Maidment C, Ahn M, Ingham B, Hinkley S, Norris G. Molecular and structural insights into skin collagen reveals several factors that influence its architecture. Int J Biol Macromol. 2019;128:509-20.

21. Manturova NE, Smirnova GO, Stupin VA, Silina EV. The ratio of collagen types I/III as a marker of skin aging and prognosis of aesthetic facial surgery results. J Pharm Sci. 2018;10:4.

\section{Abbreviations}

AFM : Achatina Fulica Mucous

ECM : Extracellular Matrix

MMP : Matrix Metalloproteinase

NDHFs : Normal Dermal Human Fibroblasts

PRP : Platelet Rich Plasma

qPCR : quantitative Polymerase Chain Reaction

ROS : Reactive Oxygen Stress

UVB : Ultra Violet-B

\section{Sponsors/Grants}

This work was supported by a grant from Ministry of Research, Technology and Higher Education of the Republic of Indonesia through Beasiswa Unggulan Dosen Indonesia Dalam Negeri (BUDI-DN) for the Ph.D. scholarship program (number PRJ-1375/LPDP.4 /2016).

\section{Potential Conflicts of Interests}

None

\section{Corresponding Author}

Ch. Tri Nuryana, Doctoral program of Medicine and Health, Medical Faculty, Public Health, and Nursing, Universitas Gadjah Mada, Yogyakarta, Indonesia 55281; Email: ch trinuryana@ugm.ac.id | ch3n yana@yahoo.co.id 